\title{
Probing optical resonances of silicon nanostructures using tunable-excitation Raman spectroscopy
}

Matthiae, M.; Nielsen, K.E.S.; Larroche, A.; Zhou, C.; Kristensen, A.; Raza, S.

Published in:

Optics Express

Link to article, DOI:

10.1364/oe.385088

Publication date:

2019

Document Version

Publisher's PDF, also known as Version of record

Link back to DTU Orbit

Citation (APA):

Matthiae, M., Nielsen, K. E. S., Larroche, A., Zhou, C., Kristensen, A., \& Raza, S. (2019). Probing optical resonances of silicon nanostructures using tunable-excitation Raman spectroscopy. Optics Express, 27(26), 38479-38492. https://doi.org/10.1364/oe.385088

\section{General rights}

Copyright and moral rights for the publications made accessible in the public portal are retained by the authors and/or other copyright owners and it is a condition of accessing publications that users recognise and abide by the legal requirements associated with these rights.

- Users may download and print one copy of any publication from the public portal for the purpose of private study or research.

- You may not further distribute the material or use it for any profit-making activity or commercial gain

- You may freely distribute the URL identifying the publication in the public portal 


\title{
Probing optical resonances of silicon nanostructures using tunable-excitation Raman spectroscopy
}

\author{
M. Matthiae, ${ }^{1}$ K. E. S. Nielsen,${ }^{1,2}$ A. Larroche, ${ }^{1}$ C. Zhou, ${ }^{1}$ A. \\ KRISTENSEN, ${ }^{1,3}$ (i) AND S. RAZA ${ }^{2,4}$ (i) \\ ${ }^{I}$ Department of Health Technology, Technical University of Denmark, 2800 Kongens Lyngby, Denmark \\ ${ }^{2}$ Department of Physics, Technical University of Denmark, 2800 Kongens Lyngby, Denmark \\ $3 a k r i @ d t u . d k$ \\ ${ }^{4}$ sraz@dtu.dk
}

\begin{abstract}
Optical materials with a high refractive index enable effective manipulation of light at the nanoscale through strong light confinement. However, the optical near field, which is mainly confined inside such high-index nanostructures, is difficult to probe with existing measurement techniques. Here, we exploit the connection between Raman scattering and the stored electric energy to detect resonance-induced near-field enhancements in silicon nanostructures. We introduce a Raman setup with a wavelength-tunable laser, which allows us to tune the Raman excitation wavelength and thereby identify Fabry-Pérot and Mie type resonances in silicon thin films and nanodisk arrays, respectively. We measure the optical near-field enhancement by comparing the Raman response on and off resonance. Our results show that tunableexcitation Raman spectroscopy can be used as a complimentary far-field technique to reflection measurements for nanoscale characterization and quality control. As proof-of-principle for the latter, we demonstrate that Raman spectroscopy captures fabrication imperfections in the silicon nanodisk arrays, enabling an all-optical quality control of metasurfaces.
\end{abstract}

(C) 2019 Optical Society of America under the terms of the OSA Open Access Publishing Agreement

\section{Introduction}

Advancements in optical technology are fueled by our understanding and characterization of optical materials at the nanoscale. Currently, there is a strong research focus on using highrefractive-index dielectrics, such as silicon, to control light through optical Mie resonances [1,2,3]. Mie resonances arise when materials with a high refractive index $n$ are structured on a length scale comparable to the wavelength of light inside the material $\lambda / n$, where $\lambda$ is the wavelength of light in free space. When the wavelength of light matches the Mie resonance wavelength, there is a buildup of electromagnetic field and energy inside the nanostructured material. The strong light confinement enables control of different properties of light, such as polarization, amplitude and phase. Mie resonances are therefore fundamental building blocks in constructing nanostructured dielectric surfaces with engineered optical functionalities [4]. These metasurfaces often contain multiple Mie resonances that act in concerto over extended lateral areas. As such, the collective efficacy of the metasurface rests on the performance of the individual Mie resonator, which are typically subwavelength in size and thus require near-field measurement techniques. As an additional hurdle, Mie resonances confine most of the electromagnetic field inside the nanostructure, which makes it challenging to probe them non-invasively with existing near-field measurement techniques. The near-field enhancement of the Mie resonance, from which properties such as resonance wavelength and phase can be determined, is therefore often inferred from electromagnetic simulations, and subsequently used for interpretation of features in reflectance or transmission spectra. Here, we show how far-field Raman spectroscopy - a technique primarily known for sensing [5] - can be used as a versatile characterization tool 
for high-refractive-index nanostructures. Raman spectroscopy measures inelastic scattering of photons from phonon and vibrational modes in materials and its signal strength depends dramatically on the electric near-field enhancement $[6,7,8]$. This dependency has driven research in surface-enhanced Raman spectroscopy to create surfaces that amplify the local electric field outside nanostructures for sensing of nearby molecules [9]. Instead of sensing external material, we exploit the phonons in the crystalline high-index material as a natural probe of the internal near field. This enables us to use a far-field Raman measurement system to determine the internal near-field enhancement created by Mie resonances without the need of any external or invasive probes.

Optical Mie resonances facilitate nanoscale control of both electric and magnetic components of light. This has been exploited to realize a plethora of exciting optical effects, including metasurface-based control of light [4], meta-lenses [10], structural color [11], Huygens' surfaces [12], lasing [13], directional control of emitters [14,15] and fluorescence [16], and active control of light [17]. These effects often involve structuring the high-index material on a scale significantly smaller than the wavelength of operation. For silicon, this length scale is on the order of few hundreds of nanometers for visible light. Such nanostructures can be realized with state-of-the-art nanolithography, but their subwavelength sizes are below the spatial resolution of optical microscopes, which makes their near-field characterization cumbersome. Scanning near-field optical microscopy has been used to map the external near field of such nanostructures $[18,19]$, but the technique requires a tip near the surface which perturbs the system. Optical modes of individual nanostructures have also been mapped with cathodoluminescence spectroscopy [20], but this requires the sample to be conductive. Photocurrent measurements provide insight into the absorption properties of individual Mie resonators [21], but require electrical contacts for current read-out. Thus, a near-field spectroscopy tool, which is versatile, non-invasive and does not set stringent requirements to the sample, would be advantageous for characterizing high-refractive-index nanostructures.

The intensity of Raman scattering is amplified by the internal near-field enhancement produced by Mie resonances in silicon nanoparticles [7,8,22]. This has been exploited for detecting these resonances, but also for other applications such as thermometry, crystallinity characterization and nonlinear spectroscopy $[23,24,25,26,27]$. In addition, the external near-field enhancements produced by Mie resonances in silicon nanoparticles has also been used to enhance the Raman signal from nearby material for sensing purposes [28,29,30,31,32]. However, these previous results are all based on Raman spectroscopy at a single fixed Raman excitation wavelength. Consequently, the identification of optical resonances would require the fabrication of many nanostructures of different sizes to gradually shift the resonance across the Raman excitation wavelength. This hinders the potential of Raman spectroscopy as a versatile characterization tool for detecting resonance-induced near-field enhancements. Here, we introduce a custom-built Raman setup that incorporates a tunable Raman laser, allowing us to tune the Raman excitation wavelength and thereby spectrally map out resonances in silicon nanostructures. In particular, we use tunable-excitation Raman spectroscopy to measure the internal field enhancement induced by both Fabry-Pérot resonances in thin silicon films and Mie resonances in silicon nanodisk arrays. By comparing the Raman signal on and off resonance, we are able to map out the optical resonances and the internal near-field enhancement. Inspired by the recent theoretical description of Raman scattering [7,8], we explain our experimental observations using a model based on the enhancement of the stored electric energy in the nanostructures. Our theoretical model is in excellent agreement with our measurements. By leveraging the dependence of the Raman signal on the internal near field, we also provide proof-of-concept experimental results of Raman spectroscopy as a tool for quality control of silicon nanostructures. We envision that tunable Raman spectroscopy can be scaled up for fast and non-invasive characterization and quality control of optical metasurfaces. 


\section{Results}

To benchmark tunable-excitation Raman spectroscopy, we first demonstrate that Raman spectroscopy with a fixed excitation wavelength can successfully detect resonance-induced near-field enhancements. We consider two systems with different types of optical resonances, namely, silicon thin films, which support Fabry-Pérot resonances, and silicon nanodisk arrays, which support Mie resonances. They provide a suitable introduction to resonance-enhanced Raman spectroscopy and enable a clearer understanding of the subsequent experiments with tunableexcitation Raman spectroscopy. We compare our fixed-excitation-wavelength results with earlier reports on similar systems $[7,33,34]$.

\subsection{Thin films of silicon}

A simple Fabry-Pérot resonator is formed by depositing a thin film of polycrystalline silicon on a fused silica substrate (Fig. 1(a)). We prepare films of different thicknesses and measure both reflectance and Raman spectra. The reflectance spectra are acquired over the visible range at normal incidence and Raman spectroscopy is performed with a fixed excitation wavelength of 785 $\mathrm{nm}$ (see Methods). The reflectance spectra show a dip due to light coupling to the second-order Fabry-Pérot resonance (Fig. 1(b)), which shifts to longer wavelengths with increasing film thickness (Fig. 1(c)). Note that the electric field intensity is maximum at the thin film surfaces (Fig. 1(b)), which is in contrast to the electric field minima seen in the classical Fabry-Pérot cavity formed by two nearby mirrors. By using data for the dispersive refractive index of our polycrystalline silicon film measured by ellipsometry (not shown), we find that well-known thin-film theory (see below) accurately captures the Fabry-Pérot resonance dips of the different film thicknesses (Fig. 1(c)).

The Raman spectra from the thin silicon films show a peak at a Stokes shifted wavenumber of $518 \mathrm{~cm}^{-1}$ (Fig. 1(d)), which corresponds to an emission wavelength of $818 \mathrm{~nm}$ for our excitation wavelength. The Raman peak is due to the optical phonon mode in silicon [35], and its measured energy of $518 \mathrm{~cm}^{-1}$ is a signature of the polycrystalline nature of our thin films [36]. The stable spectral position of the phonon band across the different thin films shows that local optothermal heating is weak and can be neglected [24]. We observe that the Raman peak intensity depends strongly on the film thickness. To correct for non-resonant Raman enhancements due to increased silicon thickness, the Raman intensity is scaled with the Fabry-Pérot resonance wavelength, which is proportional to the film thickness. By correlating the Raman spectra to the reflectance spectra, we find that the strongest Raman peak intensity occurs when the reflectance dip is spectrally located in between the Raman excitation and emission wavelengths (Figs. 1(c)-1(d)). In this case, the Fabry-Pérot resonance enhances both the excitation and emission processes of Raman scattering. To convert the Raman intensity into an enhancement that can be compared with theory, we must specify a reference Raman intensity. To this end, we normalize the Raman intensities to the off-resonant case (i.e., reflectance minimum of $635 \mathrm{~nm}$ ). We measure a clear eight-fold enhancement of the Raman signal (Fig. 1(e)), which is in agreement with our theoretical model, see below. Since the Fabry-Pérot resonance wavelength scales linearly with the silicon film thickness, this all-optical characterization requires no a priori knowledge of the film thickness and is thus a powerful tool for determining the near-field enhancement produced by a given resonance.

To explain our experimental observations, we now develop a theoretical model to estimate the Raman enhancement. The Raman signal stems from two incoherent processes: (i) the excitation of the phonon modes, which is enhanced by the electric field intensity inside the film, and (ii) the Stokes-shifted emission of light, which experiences a boost due to the decay rate enhancement (i.e., the Purcell factor) [8,33]. Both of the processes depend on the enhancement of the local electric field intensity $E^{2}$ at the corresponding wavelengths of excitation and emission [6]. We therefore calculate the Raman enhancement as the product of the electric field intensity inside 


\section{Optics EXPRESS}

a
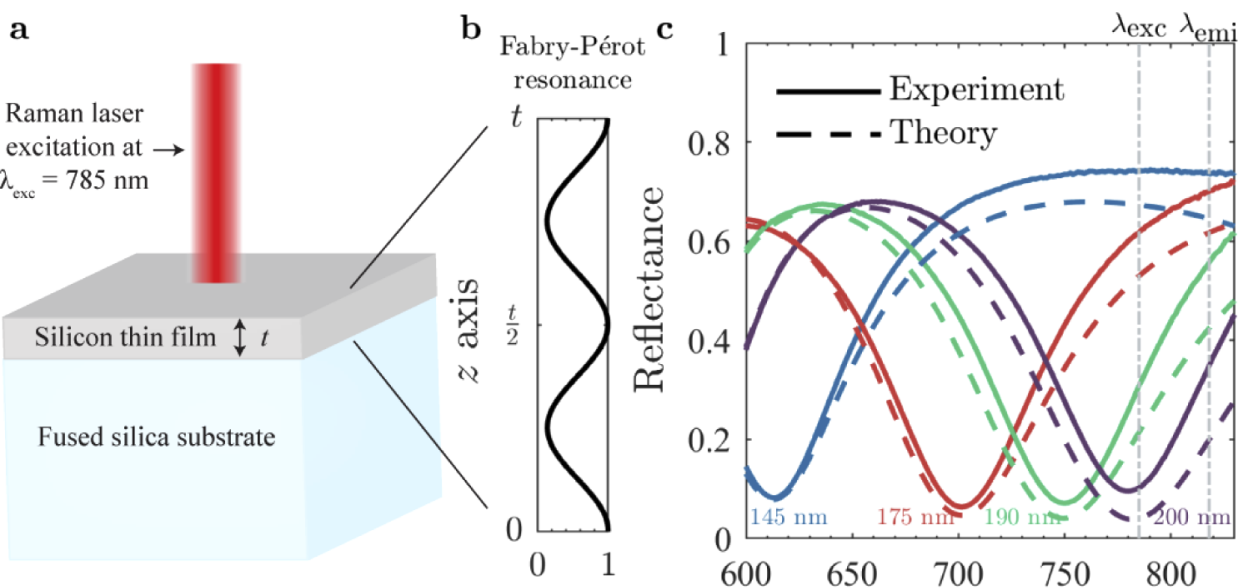

d

$|\mathbf{E}|^{2}$
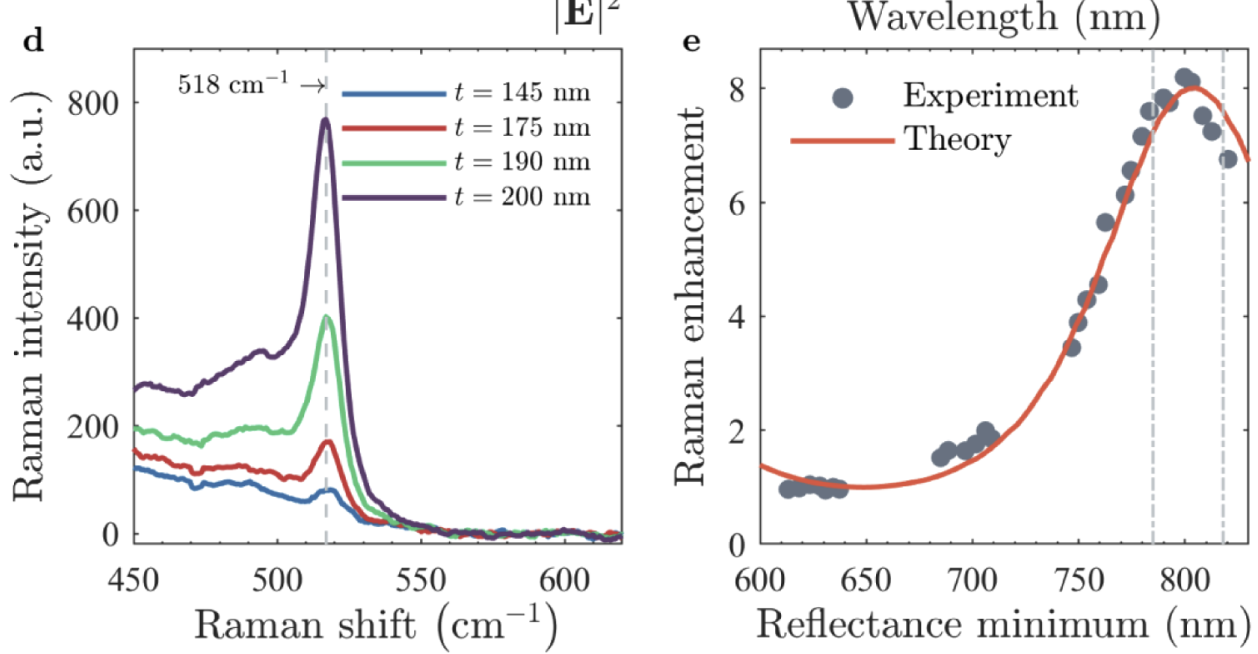

Fig. 1. Raman spectroscopy on silicon thin films. (a) A Fabry-Pérot resonator is formed by depositing a poly-crystalline silicon film of thickness $t$ on a fused silica substrate. The microscope-based Raman experiment is performed with an excitation wavelength of $785 \mathrm{~nm}$. (b) Enlarged view showing the electric-field intensity of the second-order Fabry-Pérot resonance inside the thin film. (c) Optical reflectance spectra measured on silicon films of different thicknesses. The reflectance minima, corresponding to the Fabry-Pérot resonance, have different offsets compared to the Raman excitation wavelength. (d) Raman spectra measured for different film thicknesses. The silicon Raman peak is observed at $518 \mathrm{~cm}^{-1}$ (corresponding to $818 \mathrm{~nm}$ emission wavelength) and the signal strength shows strong dependence on the film thickness. (e) Intensity of the silicon Raman peak plotted as function of wavelength of reflectance minimum and compared to the calculated Raman enhancement S. The Raman intensities of both the theoretical and experimental results are normalized to the off-resonant case (i.e., reflectance minimum of $635 \mathrm{~nm}$ ). 
the silicon film at the excitation and emission wavelengths. However, since we detect Raman signal from the entire volume of the film, we use the stored electric energy enhancement $W$ as a measure for the electric-field-intensity enhancement. This leads to the following expression for the Raman signal $S$

$$
S \propto W\left(\lambda_{\mathrm{exc}}\right) \cdot W\left(\lambda_{\mathrm{emi}}\right)
$$

where $\lambda_{\text {exc }}$ and $\lambda_{\text {emi }}$ denote the excitation and emission wavelengths, respectively. The stored electric energy enhancement is defined as $W=n^{2} \int_{V} d \mathbf{r}|\mathbf{E}|^{2} /\left(V E_{0}^{2}\right)$, where $V$ is the volume of nanostructured silicon material, $n$ is the refractive index, and $E_{0}$ is the incident electric field amplitude.

The theoretical expression for the Raman signal in Eq. (1) assumes a scalar and wavelengthindependent Raman polarizability, which is suitable for the polycrystalline silicon used in our samples. In addition, the Purcell factor is determined through the stored energy enhancement, based on the reciprocity theorem [22]. The latter is applicable to our experimental setup, which detects the Raman emission in reflectance with a low-NA objective $(\mathrm{NA}=0.3)$. Finally, the proportionality relation of Eq. (1) requires a normalization procedure for accurate comparison with experimental results. As discussed, we use the off-resonant Raman intensity as our reference intensity.

For our thin-film system, the stored electric energy enhancement is a tractable problem, which permits an analytical solution. We consider a film of thickness $t$ with a refractive index $n$ on a semi-infinite substrate with refractive index $n_{\text {sub }}$. The semi-infinite superstrate is air with a refractive index of unity. Plane-wave light of wavelength $\lambda$ is normally incident on the thin film from the air side. The incident electromagnetic wave gives rise to a reflected wave, forward- and backward-propagating waves in the film, and a transmitted wave in the substrate. By applying Maxwell's boundary conditions at both of the thin-film interfaces, we determine the electric field inside the thin film to be

$$
\mathbf{E}(z)=E_{0} \frac{t_{\mathrm{air}}}{1+r_{\mathrm{air}} r_{\mathrm{sub}} e^{i 2 k t}}\left[e^{i k z}+r_{\mathrm{sub}} e^{i 2 k t} e^{-i k z}\right] \hat{\mathbf{x}},
$$

where $t_{\text {air }}=2 /(1+n)$ and $r_{\text {air }}=(1-n) /(1+n)$ denote the Fresnel transmission and reflection at the air-silicon interface respectively, $r_{\text {sub }}=\left(n-n_{\text {sub }}\right) /\left(n+n_{\text {sub }}\right)$ denotes the Fresnel reflection at the silicon-glass interface, and $k=2 \pi n / \lambda$ is the wavenumber in the thin film. Here, the $z$-axis is defined as shown in Fig. 1(b) and the polarization is set along the $x$ direction, although this is arbitrary since we only consider normal incidence. For our experimentally relevant case of negligible material losses in the thin film, we find the stored electric energy enhancement in the thin film to be

$$
W(\lambda)=\frac{n^{2} \int_{0}^{t}|\mathbf{E}(z)|^{2} d z}{E_{0}^{2} t}=\frac{n^{2}\left|t_{\mathrm{air}}\right|^{2}}{\left|1+r_{\mathrm{air}} r_{\mathrm{sub}} e^{i 2 k t}\right|^{2}}\left[1+\left|r_{\mathrm{sub}}\right|^{2}+2\left|r_{\mathrm{sub}}\right| \operatorname{sinc}(2 k t)\right]
$$

The denominator in Eq. (3) expresses the Fabry-Pérot resonance condition, which is satisfied when the sum of the propagation and reflection phases equals an integer value of $2 \pi$. This is approximately satisfied whenever a half-integer value of wavelengths fit in the thin film.

The theoretical Raman enhancement expressed in Eqs. (1) and (3) show excellent agreement with our experimental observations (Fig. 1(e)). The model accurately captures the spectral dependence of the measured Raman enhancement and confirms that the maximum Raman enhancement occurs when the reflectance minimum (i.e., the Fabry-Pérot resonance) is located in between the Raman excitation and emission wavelengths. Our theoretical model provides physical insight into the observed Raman enhancement by linking it to the enhancement of the near field in the silicon thin film. 


\subsection{Nanodisk arrays of silicon}

Having established the link between the enhanced Raman signal and thin-film Fabry-Pérot resonances, we now turn our attention to Mie resonances that occur in nanostructured highrefractive-index materials. Mie resonances are an essential part of the design of metasurfaces, which are expected to play a significant role in future optical devices [37]. Their characterization is therefore relevant for quality control purposes, and we show here how Mie-induced near-field enhancements can be detected by Raman spectroscopy. For this task, we prepare judiciously sized silicon nanodisk arrays, which support the magnetic dipole Mie resonance. The nanodisk diameters range from $90 \mathrm{~nm}$ to $200 \mathrm{~nm}$, while the height and period are kept constant at $200 \mathrm{~nm}$ and $400 \mathrm{~nm}$, respectively (Figs. 2(a)-2(c)). These subwavelength nanodisk sizes allow us to tune the magnetic dipole resonance, which features large internal field enhancements, across our fixed Raman excitation wavelength. Reflectance measurements show two distinct peaks (Fig. 2(d)), where the peak at the longest wavelength is due to the magnetic dipole resonance of the nanodisk. The peak at the shorter wavelength corresponds to the excitation of the magnetic quadrupole resonance. Both peaks shift to longer wavelengths with increasing nanodisk diameter, but only the magnetic dipole crosses our Raman excitation wavelength. Numerical simulations of the reflectance spectra of the nanodisk array (see Methods) accurately capture the resonance wavelength of the magnetic dipole, but differ in case of the magnetic quadrupole resonance. The discrepancy in the magnetic quadrupole resonance wavelength is due to a thin residual silicon layer in our samples. Through additional simulations, we find that even a residual layer of only $10 \mathrm{~nm}$, which is within the fabrication tolerance of our samples, redshifts the magnetic quadrupole significantly (Fig. 3). Importantly, the thin residual layer has little influence on the magnetic dipole resonance, which is the mode we have access to in our Raman experiments. The magnetic dipole resonance is associated with a large stored electric energy (Fig. 2(e)), which peaks at a slightly longer wavelength than the reflectance maximum. An important feature is that the maximum stored energy enhancement decreases with increasing disk diameter. This is due to increased radiation losses, which is visible by the increase in maxima and width of the reflectance peaks. This decrease in the stored energy enhancement with increasing Mie resonance wavelength suggests that the largest Raman enhancement will occur for a particle diameter, which has its magnetic dipole resonance closer to the excitation wavelength than the emission wavelength. This is in contrast to the silicon thin film measurements (Fig. 1), where we observed the maximum Raman enhancement when the excitation and emission wavelengths were positioned symmetrically around the Fabry-Pérot resonance. The Raman measurements of the nanodisk arrays confirm our expectations (Fig. 2(f)), showing that the maximum Raman enhancement indeed occurs when the magnetic dipole resonance is close to the Raman excitation wavelength $(785 \mathrm{~nm})$. The magnetic dipole produces a remarkable 90-fold Raman enhancement, which is in good agreement with both our numerical simulations using Eq. (1) and earlier experiments on silicon nanospheres [7,34].

Besides the impressive on-resonant Raman enhancement, we also find a strong suppression of the Raman signal for off-resonant nanodisk geometries. In fact, the Raman intensity of the silicon peak for off-resonant nanodisk diameters (i.e., below $150 \mathrm{~nm}$ ) is below our detection sensitivity. As such, the Raman enhancement may be even larger than shown in Fig. 2(f). For a reasonable comparison with our simulations, we choose the reference point to be the smallest nanodisk diameter where we can detect the Raman signal from the phonon mode in silicon. This occurs for a nanodisk diameter of $150 \mathrm{~nm}$, which has the magnetic dipole resonance at a wavelength of approximately $750 \mathrm{~nm}$ (Fig. 2(f)). The simulated Raman enhancement is in good agreement with the experimental results and confirms that the maximum Raman enhancement occurs when the magnetic dipole resonance matches the Raman excitation wavelength. 


\section{Optics EXPRESS}

a

Silicon nanodisk array

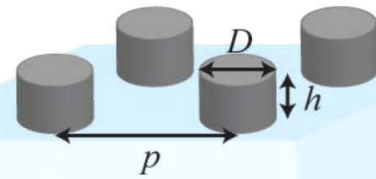

Fused silica substrate b

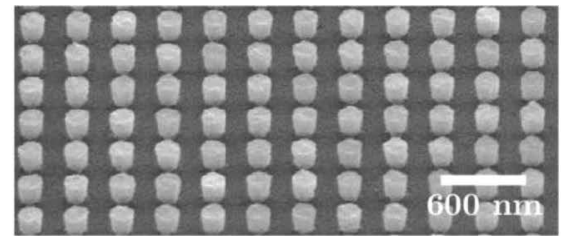

c
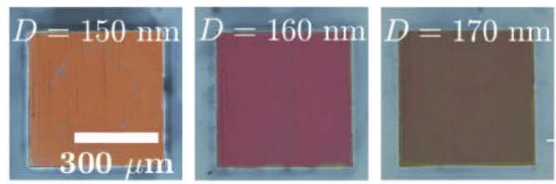
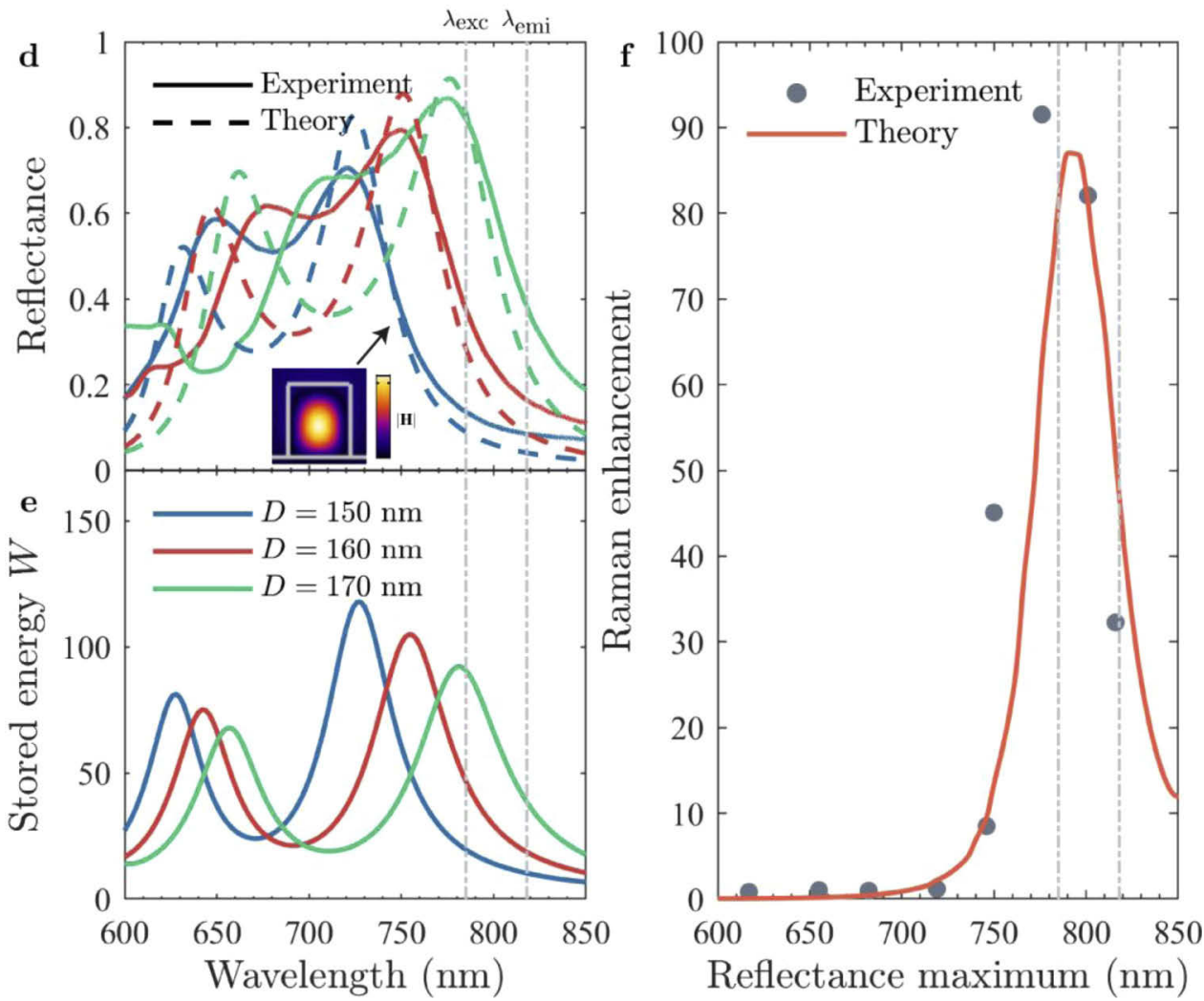

Fig. 2. Raman spectroscopy on silicon nanodisk arrays. (a) Schematic of lithographicallydefined polycrystalline silicon nanodisk arrays on a fused silica substrate. The nanodisks of diameter $D$ (ranging from $90 \mathrm{~nm}$ to $200 \mathrm{~nm}$ ) and height $h=200 \mathrm{~nm}$ form a square array of period $p=400 \mathrm{~nm}$. (b) Scanning electron microscopy and (c) optical microscopy images of the nanodisk arrays. (d) Measured and simulated reflectance spectra of nanodisk arrays of three different diameters. Inset: Magnetic field intensity of the magnetic dipole resonance. (e) Calculated stored energy enhancement spectra for the same nanodisk geometries. (f) Intensity of the silicon Raman peak plotted as function of wavelength of the magneticdipole reflectance peak. The experimental results are compared to the simulated Raman enhancement S. 


\section{Optics EXPRESS}

a

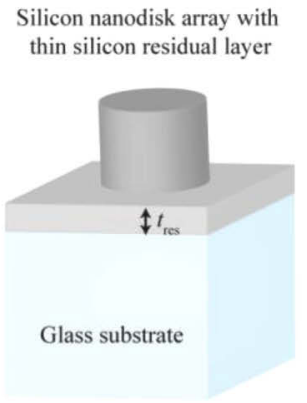

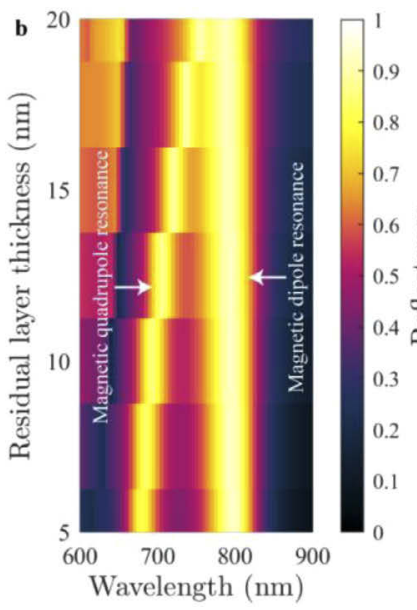

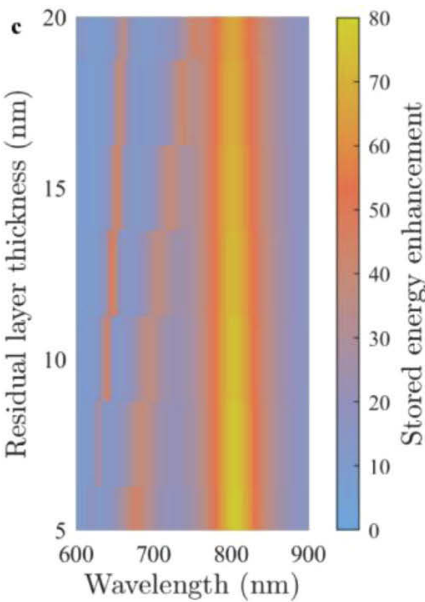

Fig. 3. Effect of thin silicon residual layer. (a) Schematic of the nanodisk unit cell with a silicon residual layer of thickness $t_{\text {res }}$. (b, c) Reflectance and stored energy enhancement spectra for different residual layer thicknesses $(D=180 \mathrm{~nm})$. The magnetic quadrupole resonance wavelength is strongly influenced by the residual layer and shifts to longer wavelengths for increasing layer thickness. Importantly, the magnetic dipole resonance wavelength and its stored energy enhancement is weakly influenced by the thin silicon layer. The stored energy peak at the shortest wavelengths is due to a Fano resonance, enabled by a coupling of the array and the localized resonance in the nanoparticle due to the thin residual layer.

\subsection{Raman spectroscopy with tunable excitation}

Our results show that Raman spectroscopy at a fixed excitation wavelength can successfully detect both Fabry-Pérot and Mie resonances in silicon nanostructures, in accordance with earlier results $[7,22,33,34]$. However, the fixed wavelength necessitates the fabrication of different samples of varying thickness and diameter to tune the resonances of interest. To alleviate this issue and increase the versatility of Raman spectroscopy, we modify our Raman setup to include a tunable Raman laser with customized filters (see Methods), which enables us to acquire Raman spectra in a range of excitation wavelengths $(760 \mathrm{~nm}$ to $810 \mathrm{~nm})$. This enables us to spectrally map a fixed resonance in detail and study the delicate interplay between the excitation wavelength and the resonance-induced Raman enhancements.

We first study the Raman enhancement produced by a fixed Fabry-Pérot resonance positioned at a wavelength of $800 \mathrm{~nm}$ corresponding to a silicon film of thickness $t=205 \mathrm{~nm}$, see Figs. 4(a)-4(c). The reflectance spectrum shows the characteristic Fabry-Pérot dip (Fig. 4(a)), which is associated with a stored energy enhancement at the exact same wavelength (Fig. 4(b)). We tune the excitation wavelength across the Fabry-Pérot resonance (Fig. 4(c)) and find that the maximum Raman intensity occurs at an excitation wavelength of approximately $785 \mathrm{~nm}$ (emission wavelength of $818 \mathrm{~nm}$ ). This places the excitation and emission wavelengths symmetrically around the Fabry-Pérot resonance. From Eq. (1), we can infer that this symmetry comes from two properties of the Fabry-Pérot resonance: (i) the resonance and its associated stored energy is spectrally symmetric, and (ii) the resonance width is much larger than the silicon phonon energy due to the low quality factor $\left(Q_{\mathrm{FP}}=4.8\right)$ of the Fabry-Pérot resonance. The former property gives rise to the symmetric Raman intensity profile as seen in Fig. 3(c), while the latter explains why the maximum Raman intensity occurs when the mean of the excitation and emission wavelengths (and not when either of them) matches the Fabry-Pérot resonance wavelength. 


\section{Optics EXPRESS}
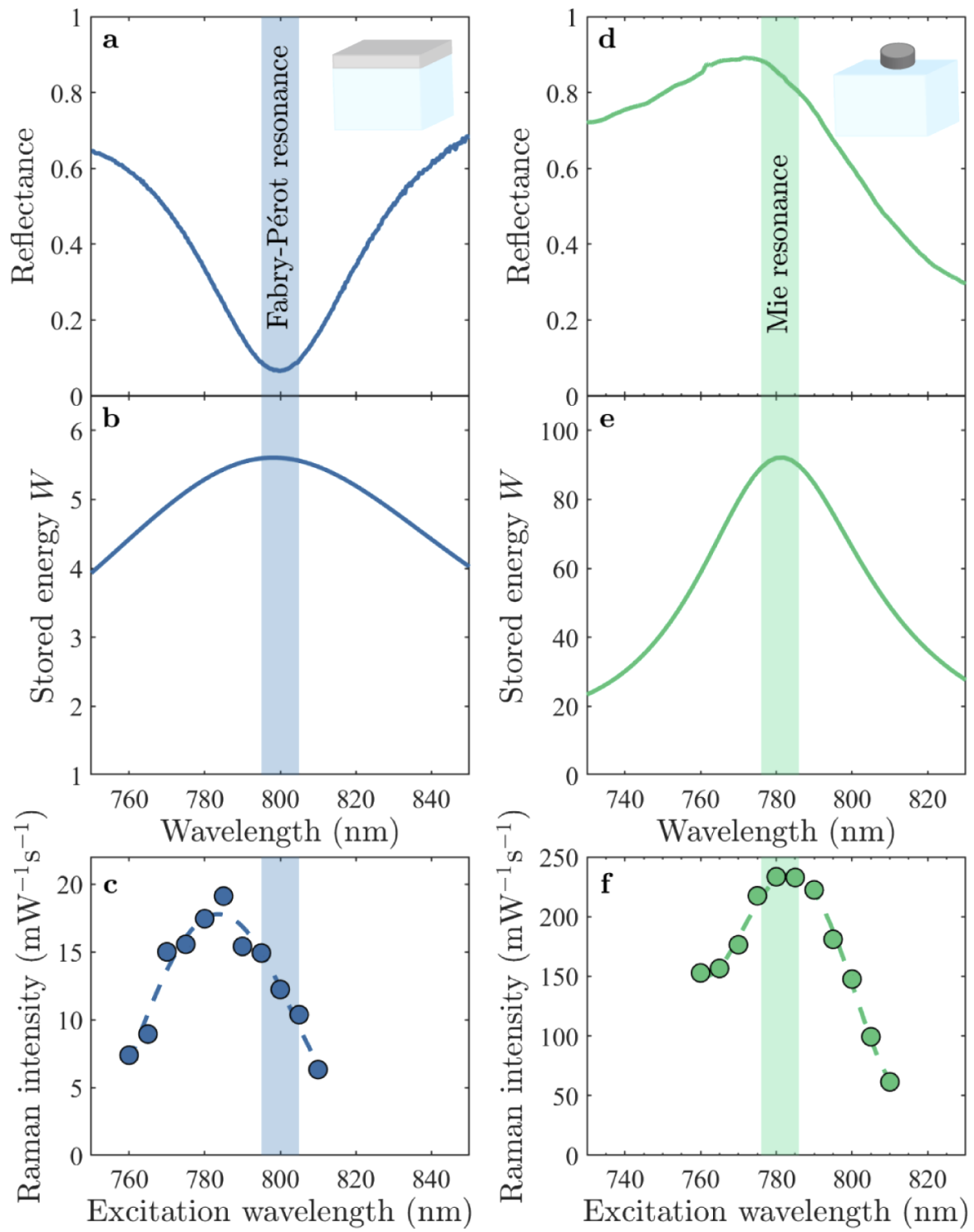

Fig. 4. Tunable excitation Raman spectroscopy. (a) Measured reflectance spectrum and (b) simulated stored energy enhancement for a silicon thin film sample with thickness $t=205 \mathrm{~nm}$, which supports a Fabry-Pérot resonance at $800 \mathrm{~nm}$. (c) Measured Raman intensity of the silicon peak as a function of excitation wavelength (with step size of $5 \mathrm{~nm}$ ). The dashed line is a guide to the eye. (d-f) Similar to (a-c) but for a nanodisk array of diameter $D=170 \mathrm{~nm}$, which supports a magnetic-dipole Mie resonance at a wavelength of $780 \mathrm{~nm}$.

This picture is different for the nanodisk array, which supports the magnetic dipole resonance (Figs. 4(d)-4(f)). Here, the reflectance spectrum shows a peak due to the magnetic dipole Mie resonance (Fig. 4(d)), with its resonance wavelength at approximately $780 \mathrm{~nm}$ as deduced from the simulated stored energy (Fig. 4(e)). By tuning the Raman excitation wavelength across the Mie resonance, we notice that the maximum Raman intensity occurs when the excitation wavelength matches the Mie resonance wavelength, which is quite different from the Fabry-Pérot case. The experimental result suggests that the excitation enhancement is significantly stronger 


\section{Optics EXPRESS}

than the emission enhancement, thus favoring the former in the Raman enhancement. Although beyond the scope of this work, we speculate that interference effects due to Mie resonances can lead to directional light scattering and emission [14,15,38], which may decrease the emission enhancement by directing the Raman emission outside the collection angle of our microscope objective. Nonetheless, Raman spectroscopy is still sensitive to the excitation enhancement, which enables us to locate the magnetic dipole resonance wavelength. The Raman measurements confirm that the magnetic dipole resonance indeed occurs at a slightly longer wavelength than the reflectance maximum, which is an important near-field insight that we would otherwise only be able to infer through simulations.

\subsection{Raman spectroscopy for quality control}

We have shown that complementing reflectance spectroscopy with Raman spectroscopy is valuable as the far and near field can be probed in the same optical system. Raman spectroscopy may therefore serve as an all-optical and non-invasive characterization tool for metasurface devices
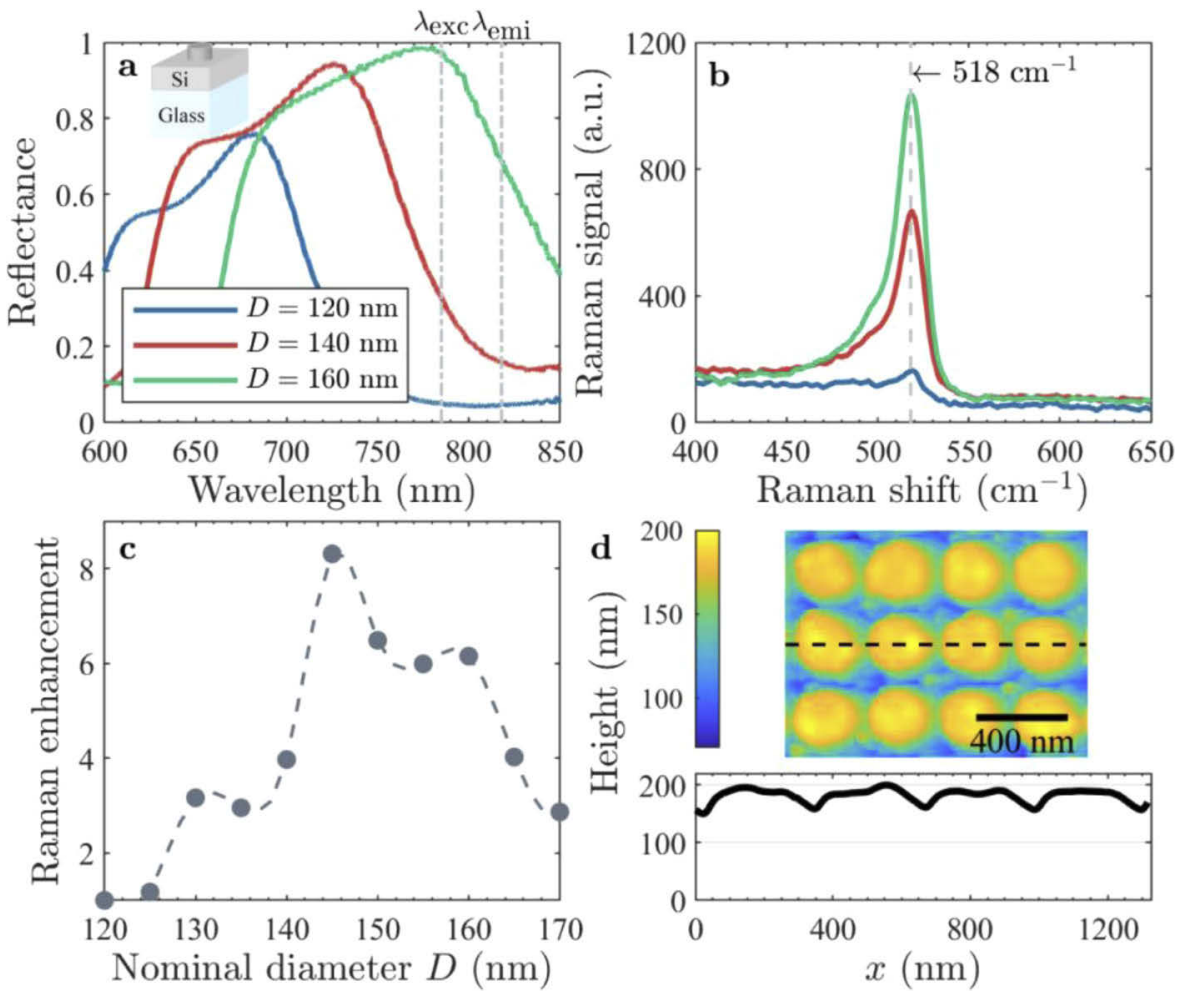

Fig. 5. Raman spectroscopy for quality control. (a) Measured reflectance and (b) Raman spectra of partially etched silicon nanodisk arrays with different nominal diameters $D$. The reflectance spectra show two peaks, which is characteristic for Mie-type optical response. Inset: Schematic of partially etched silicon nanodisks. (c) Intensity of the silicon Raman peak (at $518 \mathrm{~cm}^{-1}$ ) plotted as function of the nominal nanodisk diameter. The Raman intensities are normalized to the minimum Raman intensity measured (i.e., nominal diameter of $120 \mathrm{~nm}$ ). The Raman excitation wavelength is $785 \mathrm{~nm}$. The dashed line is a spline fit as a guide to the eye. (d) Top: Atomic-force microscopy image of the partially etched silicon nanodisk array. Bottom: Linescan of AFM image which shows that the height of the nanodisks are only around $50 \mathrm{~nm}$, instead of the designed $200 \mathrm{~nm}$ (i.e., fully etched nanodisks). 
based on crystalline high-refractive-index materials. To exemplify its potential, we consider silicon nanodisk arrays where we intentionally introduce a fabrication flaw. A critical step in the fabrication procedure is the etching procedure, which defines the nanodisk arrays. Here, we purposely disrupt the etching procedure prematurely to create ill-defined nanodisk arrays, which we expect to produce low-quality Mie resonances. We prepare partially-etched nanodisk arrays with different diameters and measure their reflectance spectra (Fig. 5(a)). Interestingly, the reflectance spectra show a double peak with slightly broader resonances than the results shown in Fig. 2(d). Based on the reflectance data alone, it is difficult to conclude that the nanodisk arrays are imperfect. Commonly, such imperfections are revealed using additional sample imaging techniques, such as atomic-force microscopy, or through comprehensive simulations, but here we offer an alternative route. By tracking the intensity of the silicon Raman peak (Figs. 5(b)-5(c)), we notice markedly different results than seen for the defect-free nanodisk arrays (Fig. 2(f)). In particular, we measure that the maximum Raman enhancement is quite weak, which suggests low quality factor Mie resonances. With both the reflectance and Raman measurements at hand, it becomes clear that these samples are faulty, which we verify using atomic-force microscopy (Fig. 5(d)). Indeed, we find that the silicon nanodisk arrays have only been partially etched, producing nanodisks with a height of only $50 \mathrm{~nm}$ placed atop a continuous silicon thin film of approximately $150 \mathrm{~nm}$ thickness. Due to the dominant thin film, this nanostructure geometry confines light only mildly below the nanodisks, producing resonances similar in quality factor to Fabry-Pérot thin-film resonances. This all-optical characterization enables a fast inspection of the fabricated sample and may potentially circumvent the need to perform time-consuming simulations to reproduce the reflectance data.

\section{Conclusion}

We have provided compelling experimental evidence that tunable-excitation Raman spectroscopy can be used to probe the optical resonances of crystalline high-refractive-index nanostructures. In particular, we have studied silicon thin films and silicon nanodisk arrays, which confine light through Fabry-Pérot and Mie resonances, respectively. These resonances produce enhancement of the near field inside the silicon, which we successfully detect with Raman spectroscopy. We have also introduced a theoretical model, which uses the stored electric energy as a measure for the Raman excitation and emission processes. Our theoretical model is in good agreement with the experimental results.

Tunable-excitation Raman spectroscopy is a far-field measurement technique, which is versatile, non-invasive and fast. Our setup used a tunable laser in the near infrared, but other tunable laser sources exist which permit to cover a broad part of the visible spectrum [39]. Combined with reflectance measurements, we have shown that Raman spectroscopy can identify faulty samples. This all-optical characterization may avoid the need for time-consuming sample imaging and comprehensive theoretical modelling. As such, Raman spectroscopy may find use as a metrology tool for inline control of future metasurface production.

\section{Methods}

\subsection{Optical setup}

A custom-built Raman microscopy setup is used in this work, which consists of three major components: a tunable near-infrared laser for optical excitation (DL Pro, Toptica Photonics, Munich), an inverted microscope (Nikon-Ti, Japan) for inspection of the sample surface and a Shamrock 303i spectrometer equipped with a deep-cooled Newton 920 CCD camera (Andor Technology, Northern Ireland) for Raman analysis and corresponding broadband reflectance measurements. All spectra are acquired with a 10x objective $(\mathrm{NA}=0.3)$ in focus with the nanostructured sample surface. 
The tunable diode laser enables sweeps of the Raman excitation wavelength from $760 \mathrm{~nm}$ to $810 \mathrm{~nm}$. For effective laser tail suppression, the cut-off wavelength of a band-pass filter needs to be tuned to go hand in hand with the actual laser wavelength in Raman spectroscopy. In order to cover the entire wavelength range with one laser line filter, a tunable band-pass filter (BP01-796/12, Semrock, New York) is installed in the optical path and its upper cut-off wavelength can be tuned by adjusting its angle with respect to the optical axis. The tunable laser line filter is accomplished by a Raman edge filter, either designed for $785 \mathrm{~nm}$ or for $808 \mathrm{~nm}$ Raman experiments. With these two exchangeable Raman edge filters and the tunable band-pass filter for the laser line, Raman experiments on polysilicon (at the wavenumber $518 \mathrm{~cm}^{-1}$ ) can be performed at all laser wavelengths in the range from $760 \mathrm{~nm}$ to $810 \mathrm{~nm}$. In practice, the sample is mounted at a fixed position while the laser wavelength is tuned. This fixed alignment allows a comparability in Raman intensity measurements. Power changes at different laser wavelengths are considered by reference measurements of the respective laser power. The maximum laser intensity in our measurements is approximately $9 \mathrm{~mW} / \mu^{2} \mathrm{~m}^{2}$, which is modest compared to the intensities required for laser-induced reshaping [24]. The low-NA objective used for focusing the Raman laser provides a beam area of approximately $9 \mu^{2}$, which means that on average 55 silicon nanodisks are illuminated.

\subsection{Data analysis}

Reflectance data are background subtracted and then normalized to the intensity of the lamp (acquired using a silver mirror, Thorlabs PF10-03-P01). All Raman data are corrected for dark counts. The Raman data from the thin film samples are further background corrected to ensure a similar Raman signal at wavelengths after the silicon peak. Each data set is normalized with respect to the Fabry-Pérot resonance wavelength (which scales linearly with the film thickness) to correct for non-resonant Raman enhancements due to increased silicon volume. To evaluate the near-field enhancement, the silicon Raman peak signal is normalized to the off-resonant case (thin film sample with resonance wavelength of $635 \mathrm{~nm}$ ). The Raman data from the nanodisk array is normalized to the nanodisk sample with a resonance at $750 \mathrm{~nm}$, where a peak is still observed. Nanodisk arrays with smaller diameters completely suppress the silicon Raman signal and therefore lead to unrealistic large enhancement factors.

\subsection{Sample fabrication}

The thin film samples are prepared by depositing polycrystalline silicon on fused silica four inch wafers using a low pressure chemical vapour deposition (LPCVD) oven at a temperature of 630 ${ }^{\circ} \mathrm{C}$. The deposition time determines the thickness of the resulting film (deposition rate, $9.5 \mathrm{~nm}$ pr. $\min )$.

The nanodisk array samples follow the same initial step as the thin film sample. To avoid interference effect in the optical measurements, the silicon layer on the backside of the wafer is removed by reactive ion etching. Afterwards positive e-beam resist (CSAR) is spin coated onto the silicon film followed by the deposition of a thin aluminum layer to avoid surface charging. Electron beam lithography is performed with a $100 \mathrm{kV}$ JEOL JBX-9500FSZ. The aluminum layer is then stripped and the resist is developed. A second aluminum layer is deposited (40 $\mathrm{nm})$, followed by a lift-off process which removes the remaining e-beam resist and leaves disk-shaped islands of aluminum. With the aluminum as an etch mask, the silicon is etched (reactive ion etcher, DRIE Pegasus) to define the nanodisk arrays. Finally, the aluminum etch mask is removed.

\subsection{Numerical simulations}

Simulations of the reflectance and stored electric energy spectra of the nanodisk arrays are performed using the finite-element software COMSOL Multiphysics 5.4. A single unit cell is simulated using periodic boundary conditions and a port in the superstrate (air domain) 
is used to send in a linearly polarized plane wave at normal incidence. We use interpolated ellipsometry-measured data for the refractive index of silicon, while the refractive index of glass is taken to be constant at 1.45 . The stored electric energy is evaluated by integrating the electric energy density over the volume of the silicon nanodisk. To determine the enhancement, we normalize the stored electric energy to the stored energy in the same volume $V$ in free space: $W_{0}=\int_{V} \mathrm{~d} V \frac{1}{4} \varepsilon_{0}\left|E_{0}\right|^{2}$, where $E_{0}$ is the amplitude of the incident plane wave. The simulated data is then multiplied by the volume of the nanodisk to make accurate comparison with experiments. As the volume of the nanodisk arrays is exactly known, it is more accurate to multiply the simulated data than divide the experimental data.

\section{Funding}

Independent Research Funding Denmark (Grant no. 7026-00117B).

\section{Acknowledgment}

We thank Professor Uriel Levy and Professor N. Asger Mortensen for fruitful discussions.

\section{Disclosures}

The authors declare no conflicts of interest.

\section{References}

1. A. I. Kuznetsov, A. E. Miroshnichenko, M. L. Brongersma, Y. S. Kivshar, and B. Luk'yanchuk, "Optically resonant dielectric nanostructures," Science 354(6314), aag2472 (2016).

2. I. Staude and J. Schilling, "Metamaterial-inspired silicon nanophotonics," Nat. Photonics 11(5), 274-284 (2017).

3. S. Jahani and Z. Jacob, "All-dielectric metamaterials," Nat. Nanotechnol. 11(1), $23-36$ (2016).

4. A. Arbabi, Y. Horie, M. Bagheri, and A. Faraon, "Dielectric metasurfaces for complete control of phase and polarization with subwavelength spatial resolution and high transmission," Nat. Nanotechnol. 10(11), 937-943 (2015).

5. P. L. Stiles, J. A. Dieringer, N. C. Shah, and R. P. Van Duyne, "Surface-enhanced Raman spectroscopy," Annu. Rev. Anal. Chem. 1(1), 601-626 (2008).

6. M. Moskovits and J. S. Suh, "Surface selection rules for surface-enhanced Raman spectroscopy: Calculations and application to the surface-enhanced Raman Spectrum of Phthalazine on Silver," J. Phys. Chem. 88(23), 5526-5530 (1984).

7. P. A. Dmitriev, D. G. Baranov, V. A. Milichko, S. V. Makarov, I. S. Mukhin, A. K. Samusev, A. E. Krasnok, P. A. Belov, and Y. S. Kivshar, "Resonant Raman scattering from silicon nanoparticles enhanced by magnetic response," Nanoscale 8(18), 9721-9726 (2016).

8. K. Frizyuk, M. Hasan, A. Krasnok, A. Alú, and M. Petrov, "Enhancement of Raman scattering in dielectric nanostructures with electric and magnetic Mie resonances," Phys. Rev. B 97(8), 085414 (2018).

9. K. Kneipp, Y. Wang, H. Kneipp, L. T. Perelman, I. Itzkan, R. R. Dasari, and M. S. Feld, "Single molecule detection using surface-enhanced Raman scattering (SERS)," Phys. Rev. Lett. 78(9), 1667-1670 (1997).

10. M. Khorasaninejad, W. T. Chen, R. C. Devlin, J. Oh, A. Y. Zhu, and F. Capasso, "Metalenses at visible wavelengths: Diffraction-limited focusing and subwavelength resolution imaging," Science 352(6290), 1190-1194 (2016).

11. A. Kristensen, J. K. W. Yang, S. I. Bozhevolnyi, S. Link, P. Nordlander, N. J. Halas, and N. A. Mortensen, "Plasmonic colour generation," Nat. Rev. Mater. 2(1), 16088 (2017).

12. M. Decker, I. Staude, M. Falkner, J. Dominguez, D. N. Neshev, I. Brener, T. Pertsch, and Y. S. Kivshar, "High-efficiency dielectric Huygens' surfaces," Adv. Opt. Mater. 3(6), 813-820 (2015).

13. S. T. Ha, Y. H. Fu, N. K. Emani, Z. Pan, R. M. Bakker, R. Paniagua-Domínguez, and A. I. Kuznetsov, "Directional lasing in resonant semiconductor nanoantenna arrays," Nat. Nanotechnol. 13(11), 1042-1047 (2018).

14. A. F. Cihan, A. G. Curto, S. Raza, P. G. Kik, and M. L. Brongersma, "Silicon Mie resonators for highly directional light emission from monolayer MoS2," Nat. Photonics 12(5), 284-290 (2018).

15. A. Vaskin, J. Bohn, K. E. Chong, T. Bucher, M. Zilk, D.-Y. Choi, D. N. Neshev, Y. S. Kivshar, T. Pertsch, and I. Staude, "Directional and spectral shaping of light emission with Mie-resonant silicon nanoantenna arrays," ACS Photonics 5(4), 1359-1364 (2018).

16. R. Regmi, J. Berthelot, P. M. Winkler, M. Mivelle, J. Proust, F. Bedu, I. Ozerov, T. Begou, J. Lumeau, H. Rigneault, M. F. García-Parajó, S. Bidault, J. Wenger, and N. Bonod, "All-dielectric silicon nanogap antennas to enhance the fluorescence of single molecules," Nano Lett. 16(8), 5143-5151 (2016).

17. A. L. Holsteen, S. Raza, P. Fan, P. G. Kik, and M. L. Brongersma, "Purcell effect for active tuning of light scattering from semiconductor optical antennas," Science 358(6369), 1407-1410 (2017). 


\section{Optics EXPRESS}

18. R. M. Bakker, D. Permyakov, Y. F. Yu, D. Markovich, R. Paniagua-Domínguez, L. Gonzaga, A. Samusev, Y. Kivshar, B. Luk'yanchuk, and A. I. Kuznetsov, "Magnetic and electric hotspots with silicon nanodimers," Nano Lett. 15(3), 2137-2142 (2015).

19. A. Y. Frolov, N. Verellen, J. Li, X. Zheng, H. Paddubrouskaya, D. Denkova, M. R. Shcherbakov, G. A. E. Vandenbosch, V. I. Panov, P. van Dorpe, A. A. Fedyanin, and V. V. Moshchalkov, "Near-field mapping of optical Fabry-Perot modes in all-dielectric nanoantennas," Nano Lett. 17(12), 7629-7637 (2017).

20. T. Coenen, J. van de Groep, and A. Polman, "Resonant modes of single silicon nanocavities excited by electron irradiation," ACS Nano 7(2), 1689-1698 (2013).

21. L. Cao, J. S. White, J.-S. Park, J. A. Schuller, B. M. Clemens, and M. L. Brongersma, "Engineering light absorption in semiconductor nanowire devices," Nat. Mater. 8(8), 643-647 (2009).

22. D. G. Baranov, R. Verre, P. Karpinski, and M. Käll, "Anapole-enhanced intrinsic Raman scattering from silicon nanodisks," ACS Photonics 5(7), 2730-2736 (2018).

23. P. A. Dmitriev, S. V. Makarov, V. A. Milichko, I. S. Mukhin, A. S. Gudovskikh, A. A. Sitnikova, A. K. Samusev, A. E. Krasnok, and P. A. Belov, "Laser fabrication of crystalline silicon nanoresonators from an amorphous film for low-loss all-dielectric nanophotonics," Nanoscale 8(9), 5043-5048 (2016).

24. M. Aouassa, E. Mitsai, S. Syubaev, D. Pavlov, A. Zhizhchenko, I. Jadli, L. Hassayoun, G. Zograf, S. Makarov, and A. Kuchmizhak, "Temperature-feedback direct laser reshaping of silicon nanostructures," Appl. Phys. Lett. 111(24), 243103 (2017).

25. G. P. Zograf, M. I. Petrov, D. A. Zuev, P. A. Dmitriev, V. A. Milichko, S. V. Makarov, and P. A. Belov, "Resonant nonplasmonic nanoparticles for efficient temperature-feedback optical heating," Nano Lett. 17(5), 2945-2952 (2017).

26. V. A. Milichko, D. A. Zuev, D. G. Baranov, G. P. Zograf, K. Volodina, A. A. Krasilin, I. S. Mukhin, P. A. Dmitriev, V. V. Vinogradov, S. V. Makarov, and P. A. Belov, "Metal-dielectric nanocavity for real-time tracing molecular events with temperature feedback," Laser Photonics Rev. 12(1), 1700227 (2018).

27. A. Krasnok, M. Tymchenko, and A. Alù, "Nonlinear metasurfaces: a paradigm shift in nonlinear optics," Mater. Today 21(1), 8-21 (2018).

28. M. Caldarola, P. Albella, E. Cortés, M. Rahmani, T. Roschuk, G. Grinblat, R. F. Oulton, A. V. Bragas, and S. A. Maier, "Non-plasmonic nanoantennas for surface enhanced spectroscopies with ultra-low heat conversion," Nat. Commun. 6(1), 7915 (2015).

29. Z. Huang, J. Wang, Z. Liu, G. Xu, Y. Fan, H. Zhong, B. Cao, C. Wang, and K. Xu, "Strong-field-enhanced spectroscopy in silicon nanoparticle electric and magnetic dipole resonance near a metal surface," J. Phys. Chem. C 119(50), 28127-28135 (2015)

30. Y.-C. Tseng, T.-Y. Lin, Y.-C. Lee, C.-K. Ku, C.-W. Chen, and H.-L. Chen, "Magnetic dipole resonance and coupling effects directly enhance the Raman signals of as-grown graphene on copper foil by over one hundredfold," Chem. Mater. 30(5), 1472-1483 (2018).

31. I. Alessandri and J. R. Lombardi, "Enhanced Raman scattering with dielectrics,” Chem. Rev. 116(24), 14921-14981 (2016).

32. A. Krasnok, M. Caldarola, N. Bonod, and A. Alú, "Spectroscopy and biosensing with optically resonant dielectric nanostructures," Adv. Opt. Mater. 6(5), 1701094 (2018).

33. K. F. McCarty, "Raman scattering as a technique of measuring film thickness: interference effects in thin growing films," Appl. Opt. 26(20), 4482-4486 (1987).

34. D. V. Murphy and S. R. J. Brueck, "Enhanced Raman scattering from silicon microstructures," Opt. Lett. 8(9), 494-496 (1983).

35. J. H. Parker Jr, D. W. Feldman, and M. Askin, "Raman scattering by silicon and germanium," Phys. Rev. 155(3), 712-714 (1967).

36. A. T. Voutsas, M. K. Hatalis, J. Boyce, and A. Chiang, "Raman spectroscopy of amorphous and microcrystalline silicon films deposited by low-pressure chemical vapor deposition," J. Appl. Phys. 78(12), 6999-7006 (1995).

37. S. Kruk and Y. Kivshar, "Functional meta-optics and nanophotonics governed by Mie resonances," ACS Photonics 4(11), 2638-2649 (2017).

38. Y. H. Fu, A. I. Kuznetsov, A. E. Miroshnichenko, Y. F. Yu, and B. Luk'yanchuk, "Directional visible light scattering by silicon nanoparticles," Nat. Commun. 4(1), 1527 (2013).

39. N. S. Mueller, S. Juergensen, K. Höflich, S. Reich, and P. Kusch, "Excitation-tunable tip-enhanced Raman spectroscopy,” J. Phys. Chem. C 122(49), 28273-28279 (2018). 